\title{
Can Tenofovir diphosphate be a candidate drug for Sars-Cov2? First clinical perspective
}

\author{
Omer Kutlu ${ }^{1}$ \\ ${ }^{1}$ Usak University, School of Medicine
}

October 1,2020

\begin{abstract}
COVID-19 pandemic continues to spread across the world in late September 2020. To date, total cases of COVID-19 exceed 33 million including 996.342 deaths according to the WHO data. Although hydroxychloroquine, oseltamivir, remdesivir, favipiravir have been reported as an anti-SARS-CoV-2 effect, it is still unclear the fully effective protective drug and treatment. Herein, we report a woman under Tenofovir diphosphate who live and close contact with his COVID-19 positive husband. Although she had close contact with his husband without measure in the home she did not show any symptoms and signs of COVID-19 and her PCR test along with antibody test negative. Given the high reproduction number of SARS-CoV-2 and long-term close contact of the case, it can be speculated that Tenofovir could interfere with the transmission of COVID-19.
\end{abstract}

\section{Can Tenofovir diphosphate be a candidate drug for Sars-Cov2? First clinical perspective}

Dear editor,

COVID-19 pandemic continues to spread across the world in late September 2020. To date, total cases of COVID-19 exceed 33 million including 996.342 deaths according to the WHO data. Governments still taking numerous measures to prevent the spread of the Severe Acute Respiratory Syndrome Coronavirus 2 (SARS-CoV-2). ${ }^{1}$ The estimate for the basic reproduction number during the early stages of the COVID-19 outbreak is recently reported as $4.22 \pm 1.69$ in Europe that shows COVID-19 has a high transmission rate. In addition, the median incubation period is reported as 6 (minimum 2, maximum 14) days. ${ }^{2}$ Although hydroxychloroquine, oseltamivir, remdesivir, favipiravir have been reported as an anti-SARS-CoV-2 effect, it is still unclear the fully effective protective drug and treatment. ${ }^{3}$

A 31-year-old man who has a high fever, chills, loss of smell, and the sore threat presented to the pandemic hospital. He had close contact history with COVID-19 patients in his workplace 6 days ago. The patient was diagnosed as COVID-19 based on the real-time reverse-transcriptase-polymerase chain reaction (RT-PCR) in nasopharyngeal swabs. He had been taken in home-quarantine and started orally 2x200 mg hydroxychloroquine. Although he had close contact with his wife without measure in the home his wife did not show any symptoms and signs of COVID-19. 18 days after the first symptoms, the antibody test was performed to the patient and his wife and her PCR test along with antibody test negative. The patient had IgG positivity while his wife did not develop antibody positivity including both IgM and IgG (Figure 1). She had been receiving Tenofovir $245 \mathrm{mg}$ daily for two years for chronic hepatitis B. She did not have any other disease as well as drug history.

SARS-CoV-2 is a positive-sense single-strand RNA virus that has RNA-dependent RNA polymerase property. ${ }^{4}$ RNA-dependent RNA polymerase is one of the main target points for the treatment of RNA viruses. Nucleotide analogs act as substrates and inhibit viral polymerases. Tenofovir diphosphate which is a nucleoside analog reverse-transcriptase inhibitor is an active form of Tenofovir alafenamide that is used 
for hepatitis B and HIV/AIDS. ${ }^{5}$ Recently, it has been shown that nucleoside analogs including 3'-fluoro-3'deoxythymidine triphosphate, 3'-azido-3'deoxythymidine triphosphate and Tenofovir diphosphate are able to terminate polymerase extension of coronavirus by consolidated with SARS-CoV-2 RNA-dependent RNA polymerase. ${ }^{6}$ Given the high reproduction number of SARS-CoV-2 and long-term close contact of the case, it can be speculated that Tenofovir could interfere with the transmission of COVID-19. In accordance with the previous in vitro study, our case is the first clinical report that supports the possible clinical protective effect of Tenofovir. Further randomized clinical studies will illuminate whether Tenofovir may be used as an effective new drug for COVID-19.

\section{References}

1. https://covid19.who.int/(accessed August 29, 2020).

2. Linla K, Peirlinck M, Kuhl E. The reproduction number of COVID-19 and its correlation with public health interventions. Comput Mech 2020;28: 1-16.

3. Shyr ZA, Gorshkov K, Z Chen CZ, Zheng W. Drug discovery strategies for SARS-CoV-2. J Pharmacol Exp Ther 2020; Jul 28. doi: 10.1124/jpet.120.000123.

4. Zumla A, Chan JF, Azhar EI, Hui DS, Yuen KY. Coronaviruses-drug discovery and therapeutic options. Nat Rev Drug Discov 2016;15: 327-47.

5. De Clercq E, Li G. Approved antiviral drugs over the past 50 years. Clin Microbiol Rev 2016;29: 695-747.

6. Chien M, Anderson TK, Jockusch S, et al. Nucleotide analogues as inhibitors of SARS-CoV-2 polymerase, a Key Drug Target for COVID-19. J Proteome Res 2020. Aug 5 doi: 10.1021/acs.jproteome.0c00392.

Figure legends

Figure 1. The patient who developed SARS-CoV-2 IgG antibody (above) and the test result of the patient's wife who receives Tenofovir (below) [Novacheck Diagnostic Kit (Colloidal Gold) for IgG/IgM antibody to SARS CoV-2]

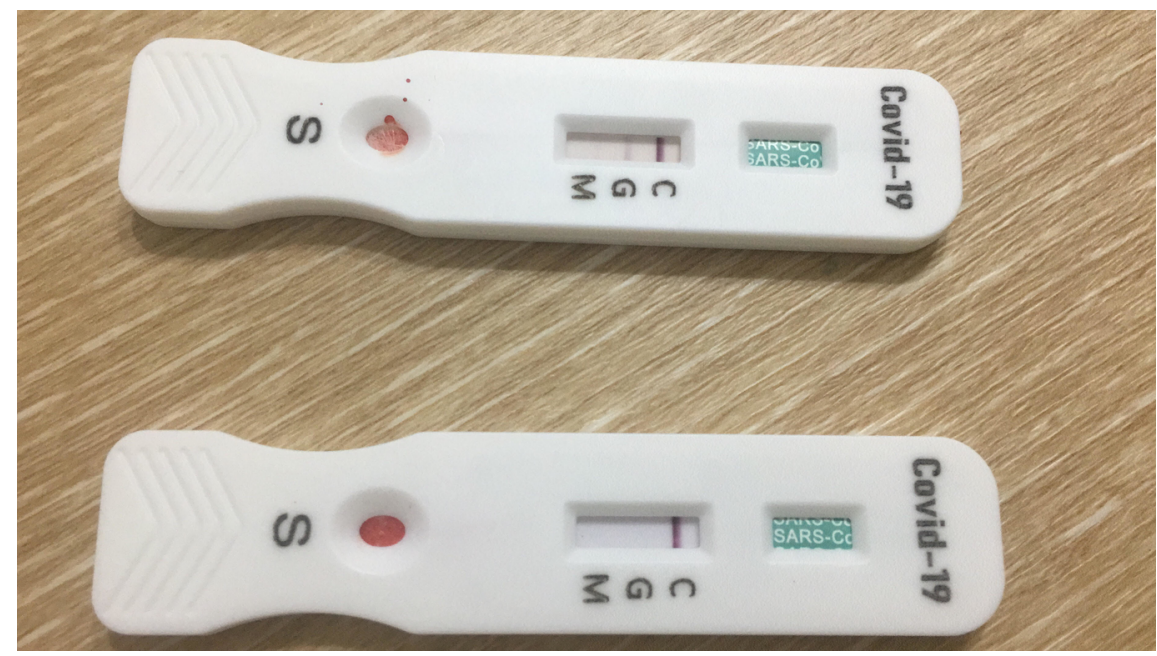

